\title{
Contamination of blood pressure cuffs by methicillin-resistant Staphylococcus aureus and preventive measures
}

\author{
M. Matsuo $\cdot$ S. Oie $\cdot$ H. Furukawa
}

Received: 20 September 2012/ Accepted: 22 April 2013/Published online: 3 May 2013

(C) The Author(s) 2013. This article is published with open access at Springerlink.com

\begin{abstract}
Background Although blood pressure cuffs are commonly used and shared in medical facilities, their routine disinfection is performed infrequently.

Aims We investigated the contamination of blood pressure cuffs by methicillin-resistant Staphylococcus aureus (MRSA).

Methods The MRSA level on the inner side (the surface in contact with patients' skin) of blood pressure cuffs used in the wards and outpatient clinics of a university hospital (733 beds) was determined using the gauze and swab wiping methods.

Results Using the gauze wiping method $(n=35)$, the MRSA contamination rate was $31.4 \%$, and the MRSA contamination level was $1,702.6 \pm 9,996.1$ (0-58, 320) colony-forming units (cfu)/cuff. No MRSA was detected on blood pressure cuffs after washing $(n=30)$ or wiping with 80 vol\% ethanol $(n=18)$.

Conclusions Blood pressure cuffs are frequently contaminated by MRSA.
\end{abstract}

Keywords Blood pressure cuff - Contamination · Methicillin-resistant Staphylococcus aureus · MRSA

\section{Introduction}

Although blood pressure cuffs are commonly used and shared in medical facilities, their routine disinfection is performed infrequently. There have been a few studies of

M. Matsuo $\cdot$ S. Oie $(\bowtie) \cdot$ H. Furukawa

Department of Pharmacy, Yamaguchi University Hospital,

1-1-1 Minamikogushi, Ube 755-8505, Japan

e-mail: oie-ygc@umin.ac.jp blood pressure cuff contamination by methicillin-resistant Staphylococcus aureus (MRSA), but in each study only a qualitative survey of MRSA was performed and the MRSA level was not described [1-3]. In addition, there are few studies on the method for maintaining the cleanliness of blood pressure cuffs [4]. Therefore, we investigated MRSA contamination of blood pressure cuffs using the swab wiping method commonly employed for the detection of contaminants [1, 3-5] and the sterile gauze wiping method [6]. Methods for maintaining the cleanliness of blood pressure cuffs were also evaluated.

\section{Materials and methods}

Quantification of MRSA on surfaces

The MRSA level on the inner side (the surface in contact with patients' skin) of blood pressure cuffs used in the wards and outpatient clinics of a university hospital (733 beds) was determined. The investigation period was from September 2009 to January 2010.

Using the swab method, the entire surface of the inner side of the blood pressure cuff was wiped using swabs moistened with sterile physiological saline. The swab samples were placed in $1.0 \mathrm{~mL}$ of nutrient broth (Nissui Pharmaceutical Co., Tokyo), stirred for $1 \mathrm{~min}$, and ultrasonicated at $36 \mathrm{kHz}$ for $5 \mathrm{~min}$. Each broth sample was diluted $10^{-1}, 10^{-2}, 10^{-3}$, and $10^{-4}$ fold in sterile saline; $0.2 \mathrm{~mL}$ of undiluted sample and of each dilution was placed onto trypticase soy agar and salt egg yolk agar (Nissui Pharmaceutical Co.) and spread with a glass hockey stick. Plates were incubated at $35^{\circ} \mathrm{C}$ for 2 days. Yellow colonies on the plates with pearl ring formation in the surrounding medium were examined by Gram-staining, 
morphological examination, the coagulase test (Staphylo La Seiken, Denka Seiken Co., Tokyo), and an Api Staph kit (Analytab Products, Plain View, New York, USA) to determine whether they were S. aureus. Staphylo La Seiken is based on the agglutination method and is composed of a latex suspension that coats human fibrinogen and rabbit IgG. The methicillin sensitivity of cultured $S$. aureus was determined using MRSA screening agar containing $6 \mathrm{mg} / \mathrm{mL}$ of oxacillin (Nippon Becton-Dickinson Co., Japan).

Sterile gauze $(15 \times 10 \mathrm{~cm})$ moistened with sterile saline was also used for wiping blood pressure cuffs and then placed in a $100-\mathrm{mL}$ bottle containing $40 \mathrm{~mL}$ of nutrient broth (Nissui Pharmaceutical Co.). The bottle was shaken for $30 \mathrm{~min}$ in a recipro-shaker (Taiyo Co., Tokyo; frequency, $250 \mathrm{~min}^{-1}$; amplitude, $50 \mathrm{~mm}$ ) and ultrasonicated (Sine Sonic 100, Ikemoto Rikagaku Co., Tokyo) at $36 \mathrm{kHz}$ for $5 \mathrm{~min}$ [7]. Each sample was diluted $10^{1}-, 10^{2}$-, and $10^{3}$ fold in sterile saline; two aliquots $(0.2 \mathrm{~mL}$ each) of each dilution and of an undiluted sample were plated on two salt egg yolk agar plates. In addition, the remaining saline sample (ca. $49 \mathrm{~mL}$ ) in the bottle was filtered through a 0.22-mm membrane filter (Nippon Becton-Dickinson Co.; diameter, $5 \mathrm{~cm}$ ), which was placed on salt egg yolk agar plates and incubated for $48 \mathrm{~h}$ at $35^{\circ} \mathrm{C}$ [8]. The methods for determining whether colonies were S. aureus and the methicillin sensitivity of cultured $S$. aureus were similar to those used for the swab method. When the membrane filtration technique revealed 50 or fewer colony-forming units (cfu) of $S$. aureus, the results obtained with this technique were used as $S$. aureus counts on the cuff surfaces. When the membrane filtration technique revealed more than about $50 \mathrm{cfu}$ (in instances where $S$. aureus could not be counted), S. aureus counts on the cuff surfaces were calculated from the diluted inocula on the egg yolk agar. When the membrane filtration technique or the inoculation onto egg yolk agar technique showed 1-9 cfu of $S$. aureus, the methicillin sensitivity of all these colonies was determined. When either of these techniques showed 10 or more cfu of $S$. aureus, ten colonies were randomly selected and their methicillin sensitivity was determined. The MRSA or methicillin-sensitive $S$. aureus count per surface was estimated from the ratio of methicillin-resistant to methicillinsensitive colonies.

Evaluation of methods to maintain cleanliness

The MRSA contamination level on a total of 30 blood pressure cuffs after outsourced washing was determined using the gauze wiping method. In addition, to evaluate the effects of wiping with ethanol for disinfection, the inner side of 18 blood pressure cuffs in use was randomly divided into halves, and one-half was wiped with 80 vol\% ethanol and left for $30 \mathrm{~min}$. The contamination level was determined using the gauze wiping method (the MRSA contamination level in the other half was used as the control).

Statistical analysis

The mean MRSA detection levels were compared by the Wilcoxon $U$ test between swab and gauze wiping method.

\section{Results}

Table 1 shows MRSA contamination of blood pressure cuffs evaluated using the swab and gauze wiping methods. The MRSA detection rate was $17.2 \%$ using the swab wiping method and $31.4 \%$ using the gauze wiping method. The MRSA contamination level was $1.9 \pm 8.8 \mathrm{cfu} / \mathrm{cuff}$ using the swab wiping method and 1,702.6 $\pm 9,996.1 \mathrm{cfu} /$ cuff using the gauze wiping method, which was significantly higher using the latter $(p<0.01)$.

None of the blood pressure cuffs $(n=30)$ after washing or after wiping with $80 \mathrm{vol} \%$ ethanol $(n=18)$ was contaminated with MRSA. However, in the controls without disinfection, the MRSA contamination rate was $22.2 \%$ and the contamination level was $9.6 \pm 36.5(1-160)$ cfu/cuff by the gauze wiping method.

\section{Discussion}

The swab method is widely used as a simple means to detect the contamination of instruments and environments due to its straightforwardness. However, the results of this study showed that the swab method is considerably inferior to the gauze wiping method for MRSA detection. Therefore, it is possible that MRSA contamination surveys underestimate contamination. In the future, the gauze wiping method should also be used in surveys of the contamination of instruments and environments.

Table 1 Contamination of blood pressure cuffs by methicillinresistant Staphylococcus aureus (MRSA) using the swab and gauze wiping method

\begin{tabular}{|c|c|c|}
\hline $\begin{array}{l}\text { Detection } \\
\text { method }\end{array}$ & $\begin{array}{l}\text { MRSA contamination } \\
\text { rate }(\%) \text { (no. of samples } \\
\text { contaminated/no. of } \\
\text { samples examined) }\end{array}$ & $\begin{array}{l}\text { Mean } \pm \text { standard } \\
\text { deviation (range) of } \\
\text { MRSA contamination } \\
\text { level (cfu/cuff) }\end{array}$ \\
\hline Swab wiping & $17.2(10 / 58)$ & $1.9 \pm 8.8(0-65)$ \\
\hline Gauze wiping & $31.4(11 / 35)$ & $\begin{array}{c}1,702.6 \pm 9,996.1 \\
(0-58,320)\end{array}$ \\
\hline
\end{tabular}

$\overline{{ }^{a}}$ The colony-forming units (cfu)/inner side of blood pressure cuff (surface in contact with patients' skin) 
Blood pressure cuffs are shared instruments, but at present may not be washed or disinfected regularly. Regular washing or disinfection of blood pressure cuffs was performed in only one ward of the dermatological department among 14 wards and outpatient clinics of the university hospital investigated in this study. In this ward of the dermatological department, blood pressure cuffs were washed at 7-day intervals. The cuffs from this ward had a lower level of MRSA contamination.

At the Japanese hospital in the fiscal year 2009, the rate of MRSA transmission was 0.3 nosocomial MRSA cases per 100 hospital admissions. This investigation of contamination status using the gauze wiping method showed MRSA on 11 (31.4\%) of 35 blood pressure cuffs examined. The MRSA detection rate was therefore high. One sample showed $5.8 \times 10^{5} \mathrm{cfu}$ of MRSA. In a previous investigation of MRSA contamination of blood pressure cuffs, Walker et al. [1] reported a contamination rate of $8 \%$ using the swab method, and de Gialluly et al. [2] reported a contamination rate of $4.4 \%$ using the contact plate method. The contamination rate in this study was far higher than that found in those studies. It is possible that blood pressure cuffs contaminated by MRSA become infection sources, and the maintenance of the cleanliness of cuffs is therefore important.

Methods for maintaining the cleanliness of cuffs include the use of disposable cuffs, washing, and wiping with ethanol. Among them, disposable cuffs are expensive but will be more widely used in the future. Washing is also effective in maintaining the cleanliness of cuffs, although it damages their texture. After wiping with alcohol, a cuff cannot be used for about $30 \mathrm{~min}$, but this is a practical method for the maintenance of cleanliness. In the hospital investigated, after the high incidence of MRSA contamination of blood pressure cuffs was clarified, it was decided that they should be wiped with alcohol once daily (cuffs are wiped with alcohol each time after use by patients with
MRSA colonization/infection) and washed at 7-day to 1-month intervals. We have seen a decline in MRSA cuff contamination on our wards since we brought in the daily alcohol wipe procedure.

\section{Conflict of interest None.}

Open Access This article is distributed under the terms of the Creative Commons Attribution License which permits any use, distribution, and reproduction in any medium, provided the original author(s) and the source are credited.

\section{References}

1. Walker N, Gupta R, Cheesbrough J (2006) Blood pressure cuff: friend or foe? J Hosp Infect 63:167-169

2. de Gialluly C, Morange V, de Gialluly E et al (2006) Blood pressure cuff as a potential vector of pathogenic microorganisms: a prospective study in a teaching hospital. Infect Control Hosp Epidemiol 27:940-943

3. Layton MC, Perez M, Heald P et al (1993) An outbreak of mupirocin-resistant Staphylococcus aureus on a dermatology ward associated with an environmental reservoir. Infect Control Hosp Epidemiol 14:369-375

4. Davis C (2009) Blood pressure cuffs and pulse oximeter sensors: a potential source of cross-contamination. Australas Emerg Nurs J 12:104-109

5. Oie S, Suenaga S, Sawa A et al (2007) Association between isolation sites of methicillin-resistant Staphylococcus aureus (MRSA) in patients with MRSA-positive body sites and MRSA contamination in their surrounding environmental surfaces. Jpn J Infect Dis 60:367-369

6. Oie S, Yanagi C, Matsui $\mathrm{H}$ et al (2005) Contamination of environmental surfaces by Staphylococcus aureus in a dermatological ward and its preventive measures. Biol Pharm Bull 28:120-123

7. Jeng DK, Lin LI, Hervey LV (1990) Importance of ultrasonical conditions in recovery of microbial contamination from material surfaces. J Appl Bacteriol 68:479-484

8. Oie S, Kamiya A (1996) Survival of methicillin-resistant Staphylococcus aureus (MRSA) on naturally contaminated dry mops. J Hosp Infect 34:145-149 